\title{
On Fault-Tolerant Partition Dimension of Homogeneous Caterpillar Graphs
}

\author{
Kamran Azhar (D), Sohail Zafar $(\mathbb{D}$, and Agha Kashif $(\mathbb{D})$ \\ University of Management and Technology (UMT), Lahore, Pakistan \\ Correspondence should be addressed to Agha Kashif; kashif.khan@umt.edu.pk
}

Received 16 August 2021; Revised 13 October 2021; Accepted 5 November 2021; Published 22 November 2021

Academic Editor: Shaojian Qu

Copyright $\odot 2021$ Kamran Azhar et al. This is an open access article distributed under the Creative Commons Attribution License, which permits unrestricted use, distribution, and reproduction in any medium, provided the original work is properly cited.

\begin{abstract}
Metric-related parameters in graph theory have several applications in robotics, navigation, and chemical strata. An important such parameter is the partition dimension of graphs that plays an important role in engineering, computer science, and chemistry. In the context of chemical and pharmaceutical engineering, these parameters are used for unique representation of chemical compounds and their structural analysis. The structure of benzenoid hydrocarbon molecules is represented in the form of caterpillar trees and studied for various attributes including UV absorption spectrum, molecular susceptibility, anisotropy, and heat of atomization. Several classes of trees have been studied for partition dimension; however, in this regard, the advanced variant, the fault-tolerant partition dimension, remains to be explored. In this paper, we computed fault-tolerant partition dimension for homogeneous caterpillars $C(p ; 1), C(p ; 2)$, and $C(p ; 3)$ for $p \geq 5, p \geq 3$, and $p \geq 4$, respectively, and it is found to be constant. Further numerical examples and an application are furnished to elaborate the accuracy and significance of the work.
\end{abstract}

\section{Introduction and Basic Terminologies}

Graph theory is a widely excelling branch of mathematics that is used to model and simplify the solution of daily-life problems. Richly engaged area of research now-a-days is the application of mathematics in chemistry. Graph theory provides simple rules to obtain many qualitative predictions about the structure and reactivity of various compounds. In the chemical graph, vertices of the graph correspond to the atoms of the molecule and edges between the vertices correspond to the chemical bonds. The caterpillar graph plays an important role in chemical graph theory for studying the combinatorial and physical properties of benzenoid hydrocarbons. They have promising uses in data reduction, modeling of interactions, computational chemistry, and ordering of graphs [1]. The partition dimension for various classes of trees such as stars, caterpillars, and homogeneous firecrackers have been computed; however, the values of partition dimensions for most kind of trees are still to be solved completely $[2,3]$. Among different parameters of graph theory, partition dimension of the graph is a unique and important parameter and has applications in network discovery and verification [4], mastermind games [5], and image processing [6].

In 2000, the concept of partition dimension of the graph was initiated by Chartrand et al. [7] as another variant of the metric dimension of graphs. The metric dimension of graph was first presented by Slater [8] and later by Harary et al. [9]. Consider $\Psi$ to be a connected graph of order $n$, where $V(\Psi)$ and $E(\Psi)$ are the set of vertices and edges, respectively. If two vertices $w, z \in V(\Psi)$, then the length of shortest path between $w$ and $z$ in $\Psi$ is the distance between these vertices and is denoted by $d(w, z)$. The distance between a vertex $z$ and $J \subseteq V(\Psi)$ is defined as $\min \{d(z, y) \mid y \in J\}$ and is denoted by $d(z, J)$. For a vertex $z \in V(\Psi), N(z)$ will denote the open neighbourhood of $z$ in $\Psi$, i.e., $N(z)=\{q \in V(\Psi): q$ is adjacent to $z\}$ and a closed neighbourhood of $z$ will be denoted by $N[z]=N(z) \cup\{z\}$ [10]. Consider $\mu=\left\{z_{1}, z_{2}, \ldots, z_{t}\right\} \in V(\Psi)$ to be an ordered subset of $V(\Psi)$. The representation of $z$ with respect to $\mu$ is $t$-tuple $\left(d\left(z, z_{1}\right), d\left(z, z_{2}\right), \ldots, d\left(z, z_{t}\right)\right)$, denoted by $r(z \mid \mu)$. The subset $\mu$ is called a resolving set of $\Psi$, if representation of $z$ with respect to $\mu$ is distinct for all $z \in V(\Psi)$. The metric dimension of $\Psi$ is defined as $\min \{|\mu|: \mu$ is resolving set of $\Psi\}$ 
and is denoted by $\beta(\Psi)$. In 2000, Chartrand et al. [11] observed the application of $\beta(\Psi)$ in pharmaceutical chemistry. Zehui et al. computed the metric dimension of the families of generalized Petersen graphs in [12]. Hussain et al. studied the metric dimension of 1-pentagonal carbon nanocone networks in [13].

In 2008, Hernando et al. [14] initiated the concept of fault-tolerant metric dimension of graphs. The resolving set $\mu$ of $V(\Psi)$ is called fault-tolerant if $\mu,\{\alpha\}$ is also a resolving set for each $\alpha \in \mu$. The fault-tolerant metric dimension of $\Psi$ is the minimum cardinality of fault-tolerant resolving set $\mu$ and is denoted by $\beta^{\prime}(\Psi)$. Raza et al. presented bounds on the fault-tolerant metric dimension of three infinite families of regular graphs [15] and also computed fault-tolerant metric dimension of convex polytopes [16]. Seyedi et al. discussed fault-tolerant metric dimension of circulant graphs in [17].

Let $\Omega=\left\{\Omega_{1}, \Omega_{2}, \ldots, \Omega_{t}\right\}$ be a partition with $t$ partition classes of the vertex set of connected graph $\Psi$. The representation of vertex $z$ with respect to partition set $\Omega$ is the $t$-vector $\left(d\left(z, \Omega_{1}\right), d\left(z, \Omega_{2}\right), \ldots, d\left(z, \Omega_{t}\right)\right)$, denoted by $r(z \mid \Omega)$. The partition $\Omega$ is called a resolving partition of $\Psi$ if the representation of all vertices in $\Psi$ is different. We define the partition dimension of graph $\Psi$ as $\min \{|\Omega|: \Omega$ is resolving partition of $\Psi\}$, and it is denoted by $\operatorname{pd}(\Psi)$. In [7], Chartrand et al. characterised the graphs with partition dimension 2 or $n$. The partition dimensions for various classes of connected graphs have been obtained. For instance, some families of trees were discussed by Fredlina et al. [18] and Rodriguez et al. [19]. Amrullah studied the partition dimension problem for a subdivision of a homogeneous firecracker in [2]. Boskoro et al. [20] characterised all graphs of order $n \geq 11$, having diameter 2 with partition dimension $n-3$. Mardhaningsih provided partition dimension of a thorn of the fan graph in [21]. Monica et al. discussed the problem for certain classes of seriesparallel graphs [22]. Chu et al. provided the sharp bounds for partition dimension of convex polytopes and flower graphs [23]. Wei et al. studied the partition dimension problem for cycle-related graphs in [24]. Circulant graphs were discussed by Maritz et al. [25]. Firstly, Gary et al. and, later, Khuller et al. mentioned the computational complexity of metric dimension of general graphs [26, 27]. The partition dimension is a graph parameter akin to the concept of metric dimension, so its computation is also more complex.

The concept of fault-tolerant version of partition dimension of graphs was initiated by Salman et al. [28]. Let $\Omega=\left\{\Omega_{1}, \Omega_{2}, \ldots, \Omega_{t}\right\}$ be a partition with $t$ partition classes of the vertex set of connected graph $\Psi$. If for each pair of distinct vertices $y, z \in V(\Psi), r(y \mid \Omega)$ and $r(z \mid \Omega)$ differ by at least two places, then the partition $\Omega$ is called fault-tolerant resolving partition of $\Psi$. The fault-tolerant partition dimension of $\Psi$ is defined as $\min \{|\Omega|: \Omega$ is fault - tolerant resolving partition of $\Psi\}$ and is denoted by $\mathscr{F}(\Psi)$. In [29], Imran et al. characterised that $\mathscr{F}(\Psi)$ of all the graphs of order $n$ is $n-1$. Recently, Kamran et al. have computed the $\mathscr{F}(\Psi)$ of tadpole and necklace graphs in [30]. Asim et al. have computed $\mathscr{F}(\Psi)$ of circulant graphs with a connection set $\{1,2\}$ in [31]. In this paper, we extend this study by considering homogeneous caterpillars $C(p ; 1), C(p ; 2)$, and $C(p ; 3)$ and show that they have constant fault-tolerant partition dimension. Some basic results on $\mathscr{F}(\Psi)$ are stated as follows.

Salman et al. revealed the following basic results on $\mathscr{F}(\Omega)$.

Proposition 1 (see [32]). For $n \geq 2$,
(a) $\operatorname{pd}(\Omega) \leq \mathscr{F}(\Omega)$
(b) $\mathscr{F}(\Omega)=n$ iff $\Omega \cong K_{n}$ or $\Omega \cong K_{n}-e$

Proposition 2 (see [28]). (a) For $n \geq 2, \mathscr{F}(\Omega) \leq \beta^{\prime}(\Omega)+1$

(b) For $n \geq 3,3 \leq \mathscr{F}(\Omega) \leq n$

The remaining part of the article is structured in the following manner: Section 2 is devoted for the computation of $\mathscr{F}(C(p ; \delta))$, where $C(p ; \delta)$ is a homogeneous caterpillar. In Section 3, we have concluded the paper by giving future research direction and application showing significance of the current work.

\section{Fault-Tolerant Partition Dimension of the Homogeneous Caterpillar Graph}

A caterpillar graph is a tree having a central path with $p$ vertices $\left\{c_{1}, c_{2}, \ldots, c_{p}\right\}$. Leaves $\delta$ are pendent vertices those are attached to every vertex of the central path. If an equal number of leaf vertices are attached to each $c_{i}$ where $1 \leq i \leq p$, then caterpillar is called the homogeneous caterpillar and is denoted by $C(p ; \delta)$. The set $V(C(p ; \delta))=C \cup A_{i j}$, where $C=\left\{c_{i}: 1 \leq i \leq p\right\}$ and $A_{i j}=\left\{a_{i j}: 1 \leq i \leq p, 1 \leq j \leq \delta\right\}$, and $E(C(p ; \delta))=\left\{c_{i} c_{i+1}: 1 \leq i \leq p-1\right\} \cup\left\{c_{i} a_{i j}: 1 \leq i \leq p, 1 \leq j \leq\right.$ $\delta\}$ are the vertex set and edge set of homogeneous caterpillar $C(p ; \delta)$, respectively. The graph of homogeneous caterpillar $C(5 ; 3)$ is shown in Figure 1.

Lemma 1 (see [18]). Let $C(p ; \delta)$ be a homogeneous caterpillar with $p, \delta \geq 1$. Then, $p d C(p ; \delta)=3$ if and only if $(\delta=1$ and $p \geq 3)$ or $(\delta=2$ and $p \geq 2)$ or $(\delta=3$ and $p \leq 3)$.

Lemma 2 (see [3]). Let $C(p ; \delta)$ be a homogeneous caterpillar with any integers $p, \delta \geq 1$. Then, $p d C(p ; \delta)=4$ if and only if $(\delta=3$ and $p \geq 4)$ or $(\delta=4$ and $p \leq 4)$.

The following theorems will allow us to compute $\mathscr{F}(C(p ; \delta))$.

Theorem 1. Let $C(p ; \delta)$ be a homogeneous caterpillar. If $\delta=$ 1 and $2 \leq p \leq 4$, then $\mathscr{F}(C(p ; 1))=3$.

Proof. Let $\Omega=\left\{\Omega_{1}, \Omega_{2}, \Omega_{3}\right\}$ be a partition with 3 partition classes of the vertices of $C(p ; 1)$. We have the following:

Case (i): for $p=2$

The $r(v \mid \Omega)$ of $C(2 ; 1)$ with respect to $\Omega_{1}=\left\{c_{1}, c_{2}\right\}$, $\Omega_{2}=\left\{a_{11}\right\}$, and $\Omega_{3}=\left\{a_{21}\right\}$ is as follows: 


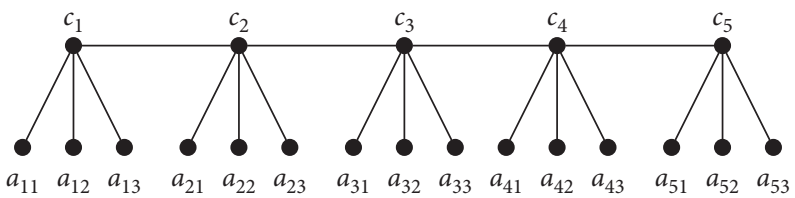

Figure 1: Homogeneous caterpillar $C(5 ; 3)$.

$$
\begin{array}{r}
r\left(c_{\sigma} \mid \Omega\right)= \begin{cases}(0,1,2), & \text { for } \sigma=1, \\
(0,2,1), & \text { for } \sigma=2,\end{cases} \\
r\left(a_{\sigma 1} \mid \Omega\right)= \begin{cases}(1,0,3), & \text { for } \sigma=1, \\
(1,3,0), & \text { for } \sigma=2 .\end{cases}
\end{array}
$$

Case (ii): for $p=3$.

Consider $\quad \Omega_{1}=\left\{c_{1}, c_{2}, a_{11}\right\}, \quad \Omega_{2}=\left\{c_{3}, a_{31}\right\}$ and $\Omega_{3}=\left\{a_{21}\right\}$. The $r(v \mid \Omega)$ of $C(3 ; 1)$ is as follows:

$$
\begin{gathered}
r\left(c_{\sigma} \mid \Omega\right)= \begin{cases}(0,2,2), & \text { for } \sigma=1, \\
(0,1,1), & \text { for } \sigma=2, \\
(1,0,2), & \text { for } \sigma=3,\end{cases} \\
r\left(a_{\sigma 1} \mid \Omega\right)= \begin{cases}(0,3,3), & \text { for } \sigma=1, \\
(1,2,0), & \text { for } \sigma=2, \\
(2,0,3), & \text { for } \sigma=3 .\end{cases}
\end{gathered}
$$

Case (iii): for $p=4$

Consider $\Omega_{1}=\left\{c_{1}, c_{2}, a_{11}\right\}, \quad \Omega_{2}=\left\{c_{3}, c_{4}, a_{41}\right\}, \quad$ and $\Omega_{3}=\left\{a_{21}, a_{31}\right\}$. The $r(v \mid \Omega)$ of $C(4 ; 1)$ is as follows:

$$
\begin{gathered}
r\left(c_{\sigma} \mid \Omega\right)= \begin{cases}(0,2,2), & \text { for } \sigma=1, \\
(0,1,1), & \text { for } \sigma=2, \\
(1,0,1), & \text { for } \sigma=3, \\
(2,0,2), & \text { for } \sigma=4,\end{cases} \\
r\left(a_{\sigma 1} \mid \Omega\right)= \begin{cases}(0,3,3), & \text { for } \sigma=1, \\
(1,2,0), & \text { for } \sigma=2, \\
(2,1,0), & \text { for } \sigma=3, \\
(3,0,3), & \text { for } \sigma=4 .\end{cases}
\end{gathered}
$$

As all the vertices have distinct representations, $\Omega$ is fault-tolerant resolving partition of $C(p ; 1)$; therefore, $\mathscr{F}(C(p ; 1)) \leq 3$. It follows from Proposition 1 (a) and Lemma 1 that $\mathscr{F}(C(p ; 1))=3$, which completes the proof.

Theorem 2. Let $C(p ; \delta)$ be a homogeneous caterpillar; if $\delta=$ 1 and $p \geq 5$, or $\delta=2$ and $p \geq 3$, then $\mathscr{F}(C(p ; \delta)) \geq 4$.

Proof. In order to prove that $\mathscr{F}(C(p ; \delta)) \geq 4$, we show that $\mathscr{F}(C(p ; \delta)) \neq 3$. Suppose on contrary that $\Omega=\left\{\Omega_{1}, \Omega_{2}, \Omega_{3}\right\}$ is a fault-tolerant partition basis of $C(p ; \delta)$. One of the partition sets $\Omega_{1}, \Omega_{2}$, or $\Omega_{3}$ contains at least one vertex of degree 3. Without loss of generality, we assume that $v$ is a vertex of degree 3 that belongs to $\Omega_{1}$, and $N(v)=\left\{z_{1}, z_{2}, z_{3}\right\}$. Suppose $\left|\Omega_{1}\right|=1$ and $N(v) \subseteq \Omega_{2} \cup \Omega_{3}$,
$\left|N(v) \cap \Omega_{2}\right| \geq 2$, or $\left|N(v) \cap \Omega_{3}\right| \geq 2$. Without loss of generality, we assume that at least two vertices $g, h \in N(v) \cap \Omega_{2}$. As $r(g \mid \Omega)=\left(1,0, e_{1}\right)$ and $r(h \mid \Omega)=\left(1,0, e_{2}\right)$ have two identical coordinates, hence a contradiction. Now, we suppose that $\left|\Omega_{1}\right| \geq 2$. We discuss the following cases:

Case 1: if $N(v) \cap \Omega_{1}=\left\{z_{1}, z_{2}, z_{3}\right\}$, then $r(v \mid \Omega)=\left(0, b_{0}, c_{0}\right), \quad r\left(z_{1} \mid \Omega\right)=\left(0, b_{1}, c_{1}\right)$, $r\left(z_{2} \mid \Omega\right)=\left(0, b_{2}, c_{2}\right)$, and $r\left(z_{3} \mid \Omega\right)=\left(0, b_{3}, c_{3}\right)$. As $b_{0}-1 \leq b_{1}, b_{2}, b_{3} \leq b_{0}+1$, by Pigeonhole principle, it is observed that two vertices have two identical coordinates in their representation, which leads to a contradiction.

Case 2: if $N(v) \cap \Omega_{1}=\left\{z_{1}, z_{2}\right\}$ and one vertex $z_{3} \in \Omega_{2}$, then $\quad r(v \mid \Omega)=\left(0,1, c_{0}\right), \quad r\left(z_{1} \mid \Omega\right)=\left(0, b_{1}, c_{1}\right)$, $r\left(z_{2} \mid \Omega\right)=\left(0, b_{2}, c_{2}\right)$, and $r\left(z_{3} \mid \Omega\right)=\left(1,0, c_{3}\right)$. Since $1 \leq b_{1}, b_{2} \leq 2$, the representation of two vertices will again be identical at two places, which is a contradiction.

Case 3: if $N(v) \cap \Omega_{1}=\left\{z_{1}\right\}$ and two vertices $z_{2}, z_{3} \in \Omega_{2}, \quad$ then $\quad r(v \mid \Omega)=\left(0,1, c_{0}\right)$, $r\left(z_{1} \mid \Omega\right)=\left(0, b_{1}, c_{1}\right), \quad r\left(z_{2} \mid \Omega\right)=\left(1,0, c_{2}\right), \quad$ and $r\left(z_{3} \mid \Omega\right)=\left(1,0, c_{3}\right)$. As $r\left(z_{2} \mid \Omega\right)$ and $r\left(z_{3} \mid \Omega\right)$ have two identical coordinates, hence a contradiction.

Case 4: if $N(v) \cap \Omega_{1}=\left\{z_{1}\right\}, z_{2} \in \Omega_{2}$ and $z_{3} \in \Omega_{3}$. We have $r(v \mid \Omega)=(0,1,1)$.

Case 4(a): for $\delta=1$ and $p \geq 5$

Consider $\quad N\left(z_{1}\right)=\left\{v, s_{1}\right\}, \quad N\left(z_{2}\right)=\{v\} \quad$ and $N\left(z_{3}\right)=\left\{v, s_{2}, s_{3}\right\}$. Let $s_{1} \in \Omega_{1}$ and $s_{2}, s_{3} \in \Omega_{3}$; then, $r\left(s_{2} \mid \Omega\right)=\left(2, q_{1}, 0\right)$ and $r\left(s_{3} \mid \Omega\right)=\left(2, q_{2}, 0\right)$, which is a contradiction. Now, if $s_{2}, s_{3} \in \Omega_{2}$, then $r\left(s_{2} \mid \Omega\right)=(2,0,1) \quad$ and $\quad r\left(s_{3} \mid \Omega\right)=(2,0,1), \quad$ a contradiction.

Case 4(b): for $\delta=2$ and $p \geq 3$

Consider, $\quad N\left(z_{1}\right)=\{v\}, \quad N\left(z_{2}\right)=\{v\}, \quad$ and $N\left(z_{3}\right)=\left\{v, s_{1}, s_{2}, s_{3}\right\}$. Let $s_{1}, s_{2}, s_{3} \in \Omega_{3} ; \quad$ then, $r\left(s_{1} \mid \Omega\right)=\left(2, q_{1}, 0\right), \quad r\left(s_{2} \mid \Omega\right)=\left(2, q_{2}, 0\right), \quad$ and $r\left(s_{3} \mid \Omega\right)=\left(2, q_{3}, 0\right)$ which leads to a contradiction. Now, let $s_{1}, s_{2} \in \Omega_{2}$ and $s_{3} \in \Omega_{3}$; then $r\left(s_{1} \mid \Omega\right)=(2,0,1)$ and $r\left(s_{2} \mid \Omega\right)=(2,0,1)$, which leads to a contradiction.

Case 5: Let $N(v) \cap \Omega_{1}=\varnothing$ and at least two vertices from $N(v)$ belong to $\Omega_{2}$. Without loss of generality, we suppose that $z_{1}, z_{2} \in \Omega_{2}$; then, $r(v \mid \Omega)=\left(0,1, c_{0}\right)$, $r\left(z_{1} \mid \Omega\right)=\left(1,0, c_{1}\right)$, and $r\left(z_{2} \mid \Omega\right)=\left(1,0, c_{2}\right)$. Again, $r\left(z_{1} \mid \Omega\right)$ and $r\left(z_{2} \mid \Omega\right)$ have two identical coordinates, which leads to a contradiction.

It is obvious from this discussion that $\mathscr{F}(C(p ; \delta)) \geq 4$, which completes the proof. 
Theorem 3. Let $C(p ; \delta)$ be a homogeneous caterpillar; if $\delta=$ 1 and $p \geq 5$, then $\mathscr{F}(C(p ; 1))=4$.

Proof. Let $\Omega=\left\{\Omega_{1}, \Omega_{2}, \Omega_{3}, \Omega_{4}\right\}$ be a partition set of $V(C(p ; 1))$. The $r(v \mid \Omega)$ of $(C(p ; 1))$, taking $\Omega_{1}=\left\{c_{i}: 1 \leq i \leq p\right\} \cup\left\{a_{11}\right\}, \quad \Omega_{2}=\left\{a_{j 1}: 2 \leq j \leq p-2\right\}$, $\Omega_{3}=\left\{a_{(p-1) 1}\right\}$, and $\Omega_{4}=\left\{a_{p 1}\right\}$, is as follows:

$$
\begin{aligned}
& r\left(c_{\sigma} \mid \Omega\right)= \begin{cases}(0,2, p-1, p), & \text { for } \sigma=1, \\
(0,1, p-\sigma, p-\sigma+1), & \text { for } 2 \leq \sigma \leq p-2, \\
(0,2,1,2), & \text { for } \sigma=p-1, \\
(0,3,2,1), & \text { for } \sigma=p,\end{cases} \\
& r\left(a_{\sigma 1} \mid \Omega\right)= \begin{cases}(0,3, p, p+1), & \text { for } \sigma=1, \\
(1,0, p+1-\sigma, p+2-\sigma), & \text { for } 2 \leq \sigma \leq p-2, \\
(1,3,0,3), & \text { for } \sigma=p-1, \\
(1,4,3,0), & \text { for } \sigma=p .\end{cases}
\end{aligned}
$$

Distinct representations of vertices of $C(p ; 1)$ show that $\Omega$ is a fault-tolerant resolving partition of $C(p ; 1)$; therefore, $\mathscr{F}(C(p ; 1)) \leq 4$. Also, by Theorem $2, \mathscr{F}(C(p ; 1)) \geq 4$, which completes the proof.

Theorem 4. Let $C(p ; \delta)$ be a homogeneous caterpillar; if $\delta=$ 2 and $p=2$, then $\mathscr{F}(C(2 ; 2))=3$.

Proof. Let $\Omega=\left\{\Omega_{1}, \Omega_{2}, \Omega_{3}\right\}$ be a partition set of $V(C(2 ; 2))$. The $r(v \mid \Omega)$ of $C(2 ; 2)$, taking $\Omega_{1}=\left\{c_{1}, a_{11}\right\}, \Omega_{2}=\left\{c_{2}, a_{21}\right\}$, and $\Omega_{3}=\left\{a_{12}, a_{22}\right\}$, is as follows:

$$
\begin{array}{r}
r\left(c_{\sigma} \mid \Omega\right)= \begin{cases}(0,1,1), & \text { for } \sigma=1, \\
(1,0,1), & \text { for } \sigma=2,\end{cases} \\
r\left(a_{\sigma 1} \mid \Omega\right)= \begin{cases}(0,2,2), & \text { for } \sigma=1, \\
(2,0,2), & \text { for } \sigma=2,\end{cases} \\
r\left(a_{\sigma 2} \mid \Omega\right)= \begin{cases}(1,2,0), & \text { for } \sigma=1, \\
(2,1,0), & \text { for } \sigma=2 .\end{cases}
\end{array}
$$

It is obvious from the representations in (5) that $\Omega$ is a fault-tolerant resolving partition of $C(2 ; 2)$; therefore, $\mathscr{F}(C(2 ; 2)) \leq 3$. It follows from Proposition 1 (a) and Lemma 1 that $\mathscr{F}(C(2 ; 2))=3$, which completes the proof.

Theorem 5. Let $C(p ; \delta)$ be a homogeneous caterpillar; if $\delta=$ 2 and $p \geq 3$, then $\mathscr{F}(C(p ; 2))=4$.

Proof. Let $\Omega=\left\{\Omega_{1}, \Omega_{2}, \Omega_{3}, \Omega_{4}\right\}$ be a partition with 4 partition classes of the vertices of $C(p ; 2)$. The $r(v \mid \Omega)$ of $C(p ; 2) \quad$ with respect to $\Omega_{1}=\left\{c_{i}: 1 \leq i \leq p\right\} \cup\left\{a_{11}, a_{i 1}: 3 \leq i \leq p-1\right\}, \Omega_{2}=\left\{a_{12}, a_{21}\right\}$, $\Omega_{3}=\left\{a_{i 2}: 2 \leq i \leq p-1\right\} \cup\left\{a_{p 1}\right\}$, and $\Omega_{4}=\left\{a_{p 2}\right\}$ is as follows:

$$
\begin{aligned}
& r\left(c_{\sigma} \mid \Omega\right)= \begin{cases}(0,1,2, p), & \text { for } \sigma=1, \\
(0, \sigma-1,1, p-\sigma+1), & \text { for } 2 \leq \sigma \leq p .\end{cases} \\
& r\left(a_{\sigma 1} \mid \Omega\right)= \begin{cases}(0,2,3, p+1), & \text { for } \sigma=1, \\
(1,0,2, p), & \text { for } \sigma=2, \\
(0, \sigma, 2, p+2-\sigma), & \text { for } 3 \leq \sigma \leq p-1, \\
(1, p, 0,2), & \text { for } \sigma=p,\end{cases} \\
& r\left(a_{\sigma 2} \mid \Omega\right)= \begin{cases}(1,0,3, p+1), & \text { for } \sigma=1, \\
(1, \sigma, 0, p+2-\sigma), & \text { for } 2 \leq \sigma \leq p-1, \\
(1, p, 2,0), & \text { for } \sigma=p .\end{cases}
\end{aligned}
$$

As all the representations are distinct, $\Omega$ is a fault-tolerant resolving partition of $C(p ; 2)$; therefore, $\mathscr{F}(C(p ; 2)) \leq 4$. Also, from Theorem $2, \mathscr{F}(C(p ; 2)) \geq 4$. This completes the proof.

Example 1. Consider the homogeneous caterpillar $C(5 ; 2)$, shown in Figure 2.

Let $\Omega=\left\{\Omega_{1}, \Omega_{2}, \Omega_{3}, \Omega_{4}\right\}$ be a partition with 4 partition classes of the vertices of $C(5 ; 2)$. The representation of vertices of $C(5 ; 2), \quad$ considering $\Omega_{1}=\left\{c_{i}: 1 \leq i \leq 5\right\} \cup\left\{a_{11}, a_{31}, a_{41}\right\}, \quad \Omega_{2}=\left\{a_{12}, a_{21}\right\}$, $\Omega_{3}=\left\{a_{22}, a_{32}, a_{42}, a_{51}\right\}$, and $\Omega_{4}=\left\{a_{52}\right\}$, is as follows:

$$
\begin{aligned}
& r\left(c_{1} \mid \Omega\right)=(0,1,2,5), \\
& r\left(c_{2} \mid \Omega\right)=(0,1,1,4), \\
& r\left(c_{3} \mid \Omega\right)=(0,2,1,3), \\
& r\left(c_{4} \mid \Omega\right)=(0,3,1,2), \\
& r\left(c_{5} \mid \Omega\right)=(0,4,1,1), \\
& r\left(a_{11} \mid \Omega\right)=(0,1,3,6), \\
& r\left(a_{12} \mid \Omega\right)=(1,0,3,6), \\
& r\left(a_{21} \mid \Omega\right)=(1,0,2,5), \\
& r\left(a_{22} \mid \Omega\right)=(1,2,0,5), \\
& r\left(a_{31} \mid \Omega\right)=(0,3,2,4), \\
& r\left(a_{32} \mid \Omega\right)=(1,3,0,4), \\
& r\left(a_{41} \mid \Omega\right)=(0,4,2,3), \\
& r\left(a_{42} \mid \Omega\right)=(1,4,0,3), \\
& r\left(a_{51} \mid \Omega\right)=(1,5,0,2), \\
& r\left(a_{52} \mid \Omega\right)=(1,5,2,0),
\end{aligned}
$$

The representations in (7) shows that $\Omega$ is a fault-tolerant resolving partition of $C(5 ; 2)$.

The following lemma will be used in computing $\mathscr{F}(C(p ; 3))$.

Lemma 3. If $C(p ; 3)$ is a homogeneous caterpillar, then we develop the relations of distances of $V(C(p ; 3))$.

Proof. The relations of distances of the vertices $C=\left\{c_{1}, c_{2}, \ldots, c_{p}\right\}$ and 


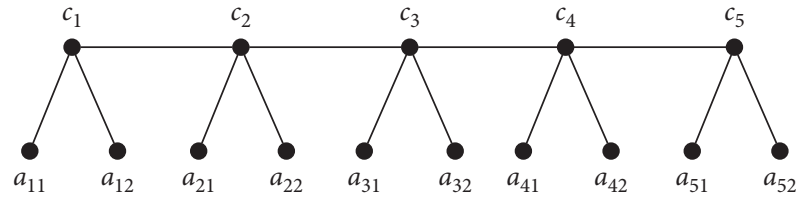

Figure 2: Homogeneous caterpillar $C(5 ; 2)$.

$A_{i 3}=\left\{a_{i 1}, a_{i 2}, a_{i 3}\right.$ : where, $\left.i \in\{1,2, \ldots, p\}\right\}$ of homogeneous caterpillar $C(p ; 3)$ are given as follows:

(a) $d\left(a_{11}, a\right)=d\left(a_{12}, a\right)=d\left(a_{13}, a\right)$, for $a \in A_{i 3}$, where $i \in\{2,3, \ldots, p\}$

(b) $d\left(a_{j 1}, a\right)=d\left(a_{j 2}, a\right)=d\left(a_{j 3}, a\right)$, for $a \in A_{13}$, where $j \in\{2,3, \ldots, p\}$

(c) $d\left(a_{31}, a\right)=d\left(a_{32}, a\right)=d\left(a_{33}, a\right)$, for $a \in A_{j 3}$, where $j \in\{2,4,5, \ldots, p\}$

(d) $d\left(a_{j 1}, c\right)=d\left(a_{j 2}, c\right)=d\left(a_{j 3}, c\right)$, for $c \in C$, where $j \in\{1,2, \ldots, p\}$

(e) $d\left(a_{21}, b\right)=d\left(a_{22}, b\right)=d\left(a_{23}, b\right)$, for $b \in C \cup A_{13}$

(f) $d\left(a_{11}, b\right)=d\left(a_{12}, b\right)=d\left(a_{13}, b\right), \quad$ for $b \in C \cup A_{t 3}, 2 \leq t \leq p$

(g) $d\left(a_{11}, b\right)=d\left(a_{12}, b\right)=d\left(a_{13}, b\right), \quad$ for $b \in C \cup \cup \cup_{i=2}^{p-1} A_{i 3}$

Theorem 6. Let $C(p ; \delta)$ be a homogeneous caterpillar; if $\delta=3$, then

$$
\mathscr{F}(C(p ; 3))= \begin{cases}4, & \text { if } 2 \leq p \leq 3 \\ 5, & \text { if } p \geq 4\end{cases}
$$

Proof. Let $\Omega=\left\{\Omega_{1}, \Omega_{2}, \Omega_{3}, \Omega_{4}\right\}$ be a partition with 4 partition classes of vertices of $C(p ; 3)$ for $p=2$ and 3 . For $p=2, \quad$ consider $\Omega_{1}=\left\{c_{1}, c_{2}, a_{11}, a_{21}\right\}, \quad \Omega_{2}=\left\{a_{12}, a_{22}\right\}$, $\Omega_{3}=\left\{a_{13}\right\}$, and $\Omega_{4}=\left\{a_{23}\right\}$. For $p=3$, consider $\Omega_{1}=\left\{c_{1}, c_{2}, c_{3}, a_{11}, a_{21}, a_{31}\right\}, \Omega_{2}=\left\{a_{12}, a_{22}\right\}, \Omega_{3}=\left\{a_{13}, a_{32}\right\}$, and $\Omega_{4}=\left\{a_{23}, a_{33}\right\}$. It can be observed easily that $\Omega$ is a faulttolerant resolving partition of $C(p ; 3)$, for $p=2$ and 3 .

Now, let $\Omega=\left\{\Omega_{1}, \Omega_{2}, \Omega_{3}, \Omega_{4}, \Omega_{5}\right\}$ be a partition with 5 partition classes of vertices of $C(p ; 3)$, for $p \geq 4$. Partition representations of the vertices of $C(p ; 3)$, taking $\Omega_{1}=\left\{c_{i}: 1 \leq i \leq p\right\} \cup\left\{a_{i 1}: 1 \leq i \leq p\right\}$,

$\Omega_{2}=\left\{a_{i 2}: 1 \leq i \leq p-1\right\}, \quad \Omega_{3}=\left\{a_{i 3}: 1 \leq i \leq p-1\right\}$, $\Omega_{4}=\left\{a_{p 2}\right\}$, and $\Omega_{5}=\left\{a_{p 3}\right\}$ are as follows:

$$
\begin{aligned}
r\left(c_{\sigma} \mid \Omega\right) & = \begin{cases}(0,1,1, p-\sigma+1, p-\sigma+1), & \text { for } 1 \leq \sigma \leq p-1, \\
(0,2,2,1,1), & \text { for } \sigma=p,\end{cases} \\
r\left(a_{\sigma 1} \mid \Omega\right) & = \begin{cases}(0,2,2, p-\sigma+2, p-\sigma+2), & \text { for } 1 \leq \sigma \leq p-1, \\
(0,3,3,2,2), & \text { for } \sigma=p,\end{cases} \\
r\left(a_{\sigma 2} \mid \Omega\right) & = \begin{cases}(1,0,2, p-\sigma+2, p-\sigma+2), & \text { for } 1 \leq \sigma \leq p-1, \\
(1,3,3,0,2), & \text { for } \sigma=p,\end{cases} \\
r\left(a_{\sigma 3} \mid \Omega\right) & = \begin{cases}(1,2,0, p-\sigma+2, p-\sigma+2), & \text { for } 1 \leq \sigma \leq p-1, \\
(1,3,3,2,0), & \text { for } \sigma=p .\end{cases}
\end{aligned}
$$

Unique representations of (9) show that $\Omega$ is a faulttolerant resolving partition of $C(p ; 3)$; therefore, $\mathscr{F}(C(p ; 3)) \leq 5$.

Now, we prove that $\mathscr{F}(C(p ; 3)) \geq 5$, for $p \geq 4$. For this, we show that $\mathscr{F}(C(p ; 3)) \neq 4$. Suppose on contrary that $\mathscr{F}(C(p ; 3))=4$. Let $\Omega=\left\{\Omega_{1}, \Omega_{2}, \Omega_{3}, \Omega_{4}\right\}$ be a partition set of $V(C(p ; 3))$ for $p \geq 4$. We discuss the following cases:

Case 1: when two partitioning sets of $\Omega$ are subsets of either $A_{i 3}: 2 \leq i \leq p$ or $C$. At least two vertices from $a_{11}, a_{12}$, and $a_{13}$ belong to the remaining two partition sets; then, by Lemma 3 ((a) and (d)), representation of two vertices will have three identical coordinates, hence a contradiction. Now, when two partitioning sets of $\Omega$ are subsets of $A_{13}$ and at least two vertices from $a_{21}, a_{22}$, and $a_{23}$ belong to the remaining two partition sets, then by Lemma 3 (b), representation of two vertices will have three identical coordinates, hence a contradiction.

Case 2: we discuss cases when two partitioning sets are a subset of union of 2 sets of vertices:

Case 2(a): when two partitioning sets of $\Omega$ are subsets of $A_{13} \cup A_{23}$ and at least two vertices from $a_{31}, a_{32}$, and $a_{33}$ belong to the remaining two partition sets, then by Lemma 3 (c), representation of two vertices will have three identical coordinates, which is a contradiction. When two partitioning sets of $\Omega$ are subsets of either $A_{13} \cup A_{t 3}: 3 \leq t \leq p$ and at least two vertices from $a_{21}, a_{22}$, and $a_{23}$ belong to the remaining two partition sets, then by Lemma 3 (b), representation of two vertices will have three identical coordinates, which is a contradiction.

Case 2(b): when two partitioning sets of $\Omega$ are subsets of either $A_{q 3} \cup A_{r 3}: 2 \leq q \leq p-1, p+1 \leq r \leq p$ and at least two vertices from $a_{11}, a_{12}$, and $a_{13}$ belong to the remaining two partition sets, then by Lemma 3 (a), representation of two vertices will have three identical coordinates, which is a contradiction.

Case 2(c): when two partitioning sets of $\Omega$ are subsets of $C \cup A_{13}$ and at least two vertices from $a_{21}, a_{22}$, and $a_{23}$ belong to the remaining two partition sets, then by Lemma 3 (e), representation of two vertices will have three identical coordinates, which is a contradiction. Finally, when two partitioning sets of $\Omega$ are subsets of either $C \cup A_{t 3}: 2 \leq t \leq p$ and at least two vertices from $a_{11}, a_{12}$, and $a_{13}$ belong to the remaining two partition sets, then by Lemma 3 (f), representation of two vertices will have three identical coordinates, which is a contradiction.

Case 3: now we discuss cases when two partitioning sets are subsets of union of $3,4, \ldots, p$ sets of vertices:

Case 3(a): when two partitioning sets of $\Omega$ are subsets of either $\cup_{i=3}^{p} A_{i 3}$, the remaining two partition sets will contain at least two $c_{i}, c_{j} \in C$. Without loss of generality, we suppose that $c_{i}, c_{j}$ belong to $\Omega_{3}$; then, $r\left(c_{i} \mid \Omega\right)=(1,1,0, s)$ and $r\left(c_{j} \mid \Omega\right)=(1,1,0, w)$, which is a contradiction. 


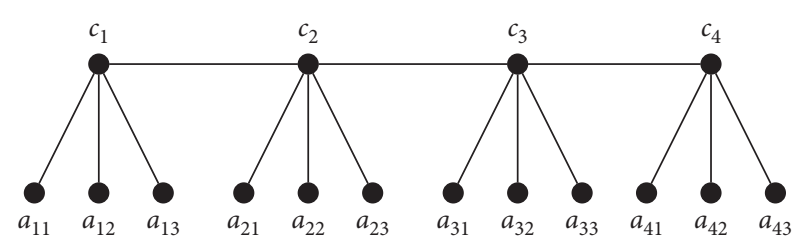

FIgURE 3: Homogeneous caterpillar $C(4 ; 3)$.

Case 3(b): when two partitioning sets of $\Omega$ are subsets of either $C \cup \cup_{i=2}^{p-1} A_{i 3}$, and if $A_{13} \notin C \cup \cup_{i=2}^{p-1} A_{i 3}$, at least two vertices from $a_{11}, a_{12}$, and $a_{13}$ belong to the remaining two partition sets, then by Lemma $3(\mathrm{~g})$, representation of two vertices will have three identical coordinates, which is a contradiction. If $A_{13} \in C \cup \cup \cup_{i=2}^{p-1} A_{i 3}$, then let $A_{e 3}$ be a vertex set not contained in $C \cup \cup \cup_{i=2}^{p-1} A_{i 3}$. At least two vertices from $a_{e 1}, a_{e 2}$, and $a_{e 3}$, belong to the remaining two partition sets. Representation of these two vertices with respect to $\Omega$ will have three identical coordinates, which is a contradiction.

Case 4: when two partition sets of $\Omega$ are subsets of $C \cup A_{13} \cup A_{23} \cup \cdots \cup A_{p 3}$, without loss of generality, we suppose these subsets belong to $\Omega_{1}$ and $\Omega_{2}$ :

Case 4(a): at least two of $a_{i 1}, a_{i 2}, a_{i 3}$ belong to one of $\Omega_{1}$ or $\Omega_{2}$. Without loss of generality, we suppose that $a_{i 1}, a_{i 2} \in \Omega_{1}$ and $a_{i 3} \in \Omega_{2}$; then, $r\left(a_{i 1} \mid \Omega\right)=(0, b, \alpha, s)$ and $r\left(a_{i 2} \mid \Omega\right)=(0, b, \alpha, s)$, where $1 \leq b \leq 2$, hence a contradiction.

Case 4(b): one of the remaining two partition sets contain at least two $c_{i}, c_{j} \in C$. Without loss of generality, we suppose that $c_{i}, c_{j}$ belong to $\Omega_{3}$; then, $r\left(c_{i} \mid \Omega\right)=(1,1,0, s)$ and $r\left(c_{j} \mid \Omega\right)=(1,1,0, w)$, which is a contradiction.

Case 4(c): neither two of $a_{i 1}, a_{i 2}, a_{i 3}$ where $1 \leq i \leq p$ belong to the same partition set, nor two of $c_{i}, c_{j}$ belong to $\Omega_{3}$ or $\Omega_{4}$. For $4 \leq p \leq 6$, at least two vertices in $\Omega_{1}$ will have representations of forms $r(0,2,2, p)$, $r(0,2, q, 2),(0,2, r, 1), r(0,2,1, s), r(0,1,2, t)$, and $r(0,1, u, 2)$, which leads to a contradiction. For $p \geq 7$, at least three vertices $c_{k}, c_{l}$, and $c_{m}$ belong to $\Omega_{1}$ or $\Omega_{2}$. Without loss of generality, we suppose these vertices belong to $\Omega_{1}$; then, $r\left(c_{k} \mid \Omega\right)=(0,1,1,-)$ or $(0,1,-, 1)$, $r\left(c_{l} \mid \Omega\right)=(0,1,1,-) \quad$ or $\quad(0,1,-, 1), \quad$ and $r\left(c_{m} \mid \Omega\right)=(0,1,1,-)$ or $(0,1,-, 1)$. Representation of at least two of $c_{k}, c_{l}$, and $c_{m}$ will be the same at three places, hence a contradiction.

The above discussion shows that $\mathscr{F}(C(p ; 3)) \geq 5$. This completes the proof.

Example 2. Consider the homogeneous caterpillar $C(4 ; 3)$, shown in Figure 3.

Let $\Omega=\left\{\Omega_{1}, \Omega_{2}, \Omega_{3}, \Omega_{4}, \Omega_{5}\right\}$ be a partition with 5 partition classes of the vertices of $C(4 ; 3)$. The representations of vertices of $\quad C(4 ; 3)$, considering $\Omega_{1}=\left\{c_{i}: 1 \leq i \leq 4\right\} \cup\left\{a_{11}, a_{21}, a_{31}, a_{41}\right\}, \quad \Omega_{2}=\left\{a_{12}, a_{22}, a_{32}\right\}$,
$\Omega_{3}=\left\{a_{13}, a_{23}, a_{33}\right\}, \quad \Omega_{4}=\left\{a_{42}\right\}$, and $\Omega_{5}=\left\{a_{43}\right\}$, are as follows:

$$
\begin{aligned}
& r\left(c_{1} \mid \Omega\right)=(0,1,1,4,4), \\
& r\left(c_{2} \mid \Omega\right)=(0,1,1,3,3), \\
& r\left(c_{3} \mid \Omega\right)=(0,1,1,2,2), \\
& r\left(c_{4} \mid \Omega\right)=(0,2,2,1,1), \\
& r\left(a_{11} \mid \Omega\right)=(0,2,2,5,5), \\
& r\left(a_{12} \mid \Omega\right)=(1,0,2,5,5), \\
& r\left(a_{13} \mid \Omega\right)=(1,2,0,5,5), \\
& r\left(a_{21} \mid \Omega\right)=(0,2,2,4,4), \\
& r\left(a_{22} \mid \Omega\right)=(1,0,2,4,4), \\
& r\left(a_{23} \mid \Omega\right)=(1,2,0,4,4), \\
& r\left(a_{31} \mid \Omega\right)=(0,2,2,3,3), \\
& r\left(a_{32} \mid \Omega\right)=(1,0,2,3,3), \\
& r\left(a_{33} \mid \Omega\right)=(1,2,0,3,3), \\
& r\left(a_{41} \mid \Omega\right)=(0,3,3,2,2), \\
& r\left(a_{42} \mid \Omega\right)=(1,3,3,0,2), \\
& r\left(a_{43} \mid \Omega\right)=(1,3,3,2,0) .
\end{aligned}
$$

The representations in (10) shows that $\Omega$ is a fault-tolerant resolving partition of $C(4 ; 3)$.

\section{Conclusion}

In this paper, we have computed that $F(C(p ; \delta))$ for $\delta=1,2$, and 3 is between 3 and 5 . The obtained results led us to the conclusion that the structures of homogeneous caterpillars $C(p ; 1), C(p ; 2)$, and $C(p ; 3)$ have constant fault-tolerant partition dimension for $p \geq 5, p \geq 3$, and $p \geq 4$, respectively. Future research can focus on computing the fault-tolerant partition dimension for the classes of homogeneous caterpillar $C(p ; \delta)$, when $\delta=4,5$, and 6 .

Here, we include an application of routing optimization problem that shows the significance of the current work. Consider a company that wants to pick passengers from different locations in a certain area using minimum resources and avoiding repeated visits. If locations are considered as nodes and roads connecting them as edges of a graph, then locations can be grouped together that require a single vehicle to pick the passenger. The minimum number of grouping required to represent each location uniquely can be realised as the partition dimension problem of the graph. Also, the minimum number of grouping required to uniquely represent each location even if one of the groups is inaccessible relates to fault-tolerant partition dimension of the graph.

\section{Data Availability}

The data used to support the findings of this study are included within the article. 


\section{Conflicts of Interest}

The authors declare that they have no conflicts of interest.

\section{References}

[1] S. El-Basil, "Caterpillar (Gutman) trees in chemical graph theory," Advances in the Theory of Benzenoid Hydrocarbons, Springer, Berlin, Heidelberg, 1990.

[2] A. Amrullah, "The partition dimension of a subdivision of a homogeneous firecracker," Electronic Journal of Graph Theory and Applications, vol. 8, no. 2, pp. 445-455, 2020.

[3] K. I. B. K. P. Arimbawa and E. T. Baskoro, "Partition dimension of some classes of trees," Procedia Computer Science, vol. 74, pp. 67-72, 2015.

[4] Z. Beerliová, F. Eberhard, T. Erlebach et al., "Network discovery and verification," IEEE Journal on Selected Areas in Communications, vol. 24, no. 12, pp. 2168-2181, 2006.

[5] V. Chvatal, "Mastermind," Combinatorica, vol. 3, no. 3-4, pp. 325-329, 1983.

[6] R. A. Melter and I. Tomescu, "Metric bases in digital geometry," Computer Vision, Graphics, and Image Processing, vol. 25, no. 1, pp. 113-121, 1984.

[7] G. Chartrand, E. Salehi, and P. Zhang, "The partition dimension of a graph," Aequationes Mathematicae, vol. 59, no. 1 , pp. $45-54,2000$.

[8] P. J. Slater, "Leaves of trees," Congruent Number, vol. 14, pp. 549-559, 1975.

[9] F. Harary and R. A. Melter, "On the metric dimension of a graph," Theory of Computing Systems, vol. 2, pp. 191-195, 1976.

[10] A. Estrada-Moreno, "On the k-partition dimension of graphs," Theoretical Computer Science, vol. 806, pp. 42-52, 2020.

[11] G. Chartrand, L. Eroh, M. A. Johnson, and O. R. Oellermann, "Resolvability in graphs and the metric dimension of a graph," Discrete Applied Mathematics, vol. 105, no. 1-3, pp. 99-113, 2000.

[12] Z. Shao, S. M. Sheikholeslami, P. Wu, and J.-B. Liu, "The metric dimension of some generalized Petersen graphs," Discrete Dynamics in Nature and Society, vol. 2018, Article ID 4531958, 10 pages, 2018.

[13] Z. Hussain, M. Munir, A. Ahmad, M. Chaudhary, J. Alam Khan, and I. Ahmed, "Metric basis and metric dimension of 1pentagonal carbon nanocone networks," Scientific Reports, vol. 10, no. 1, Article ID 19687, 2020.

[14] C. Hernando, M. Mora, P. J. Slater, and D. R. Wood, "Faulttolerant metric dimension of graphs," in Proceedings of the International Conference on Convexity in Discrete Structures, Ramanujan Mathematical Society, Tiruchirappalli, India, June 2008.

[15] H. Raza, S. Hayat, M. Imran, and X.-F. Pan, "Fault-tolerant resolvability and extremal structures of graphs," Mathematics, vol. 7, no. 1, p. 78, 2019.

[16] H. Raza, S. Hayat, and X.-F. Pan, "On the fault-tolerant metric dimension of convex polytopes," Applied Mathematics and Computation, vol. 339, pp. 172-185, 2018.

[17] N. Seyedi and H. R. Maimani, "Fault-tolerant metric dimension of circulant graphs," Facta Universitatis-Series: Mathematics and Informatics, vol. 34, no. 4, pp. 781-788, 2019.

[18] K. Q. Fredlina and E. T. Baskoro, "The partition dimension of some families of trees," Procedia Computer Science, vol. 74, pp. 60-66, 2015.
[19] J. A. Rodŕiguez-Velazquez, I. G. Yero, and M. Lemańska, "On the partition dimension of trees," Discrete Applied Mathematics, vol. 166, pp. 204-209, 2014.

[20] E. T. Baskoro and D. O. Haryeni, "All graphs of order $n \geq 11$ and diameter 2 with partition dimension $n-3$," Heliyon, vol. 6, no. 4, Article ID e03694, 2020.

[21] A. Mardhaningsih, "A note on the partition dimension of thorn of fan graph," Jurnal Matematika MANTIK, vol. 5, no. 1, pp. 45-49, 2019.

[22] C. M. Mohan, S. Santhakumar, M. Arockiaraj, and J.-B. Liu, "Partition dimension of certain classes of series parallel graphs," Theoretical Computer Science, vol. 778, pp. 47-60, 2019.

[23] Y.-M. Chu, M. F. Nadeem, M. Azeem, and M. K. Siddiqui, "On sharp bounds on partition dimension of convex polytopes," IEEE Access, vol. 8, pp. 224781-224790, 2020.

[24] C. Wei, M. F. Nadeem, H. M. A. Siddiqui, M. Azeem, J. B. Liu, and A. Khalil, "On partition dimension of some cycle-related graphs," Mathematical Problems in Engineering, vol. 2021, Article ID 4046909, 8 pages, 2021.

[25] E. C. M. Maritz and T. Vetrík, "The partition dimension of circulant graphs," Quaestiones Mathematicae, vol. 41, no. 1, pp. 49-63, 2018.

[26] M. R. Garey and D. S. Johnson, Computers and Intractability: A Guide to the Theory of NP-Completeness, Freeman, New York, NY, USA, 1979.

[27] S. Khuller, B. Raghavachari, and A. Rosenfeld, "Landmarks in graphs," Discrete Applied Mathematics, vol. 70, no. 3, pp. 217-229, 1996.

[28] M. Salman, I. Javaid, M. Chaudhry, and S. Shokat, "Faulttolerance in resolvability," Utilitas Mathematica, vol. 80, pp. 263-275, 2009.

[29] I. Javaid, M. Salman, and M. A. Chaudhry, "Graphs of order $n$ with fault-tolerant partition dimension $n-1$," UPB Scientific Bulletin, Series A: Applied Mathematics and Physics, vol. 76, no. 3, 2014

[30] K. Azhar, S. Zafar, A. Kashif, and Z. Zahid, "On fault-tolerant partition dimension of graphs," Journal of Intelligent and Fuzzy Systems, vol. 40, no. 1, pp. 1129-1135, 2021.

[31] A. Nadeem, A. Kashif, S. Zafar, and Z. Zahid, "On 2-partition dimension of the circulant graphs," Journal of Intelligent and Fuzzy Systems, vol. 40, no. 5, pp. 9493-9503, 2021.

[32] M. Salman, I. Javaid, and M. Chaudhry, "Fault-tolerant metric and partition dimension of graphs," Utilitas Mathematica, vol. 83, pp. 187-199, 2010. 